\title{
OCCUPY WALL STREET VS. LA RETÓRICA DEL MIEDO
}

\section{OCCUPY WALL STREET VS. RHETORIC OF FEAR}

\section{Henry Campos Vargas*}

\author{
Dedicado a \\ mi hermano Rafael
}

\begin{abstract}
RESUMEN
La respuesta de la Administración Obama al movimiento Occupy Wall Street es un claro ejemplo de la aplicación de la retórica del miedo. Debido a esto, el presente artículo pretende ofrecer una aproximación retórica de los textos empleados por Occupy Wall Street, y cómo estos interactuaron con la administración norteamericana.

PALABRAS CLAVE: ESTADOS UNIDOS * OCCUPY WALL STREET * RETÓRICA * MIEDO * POLÍTICA

\section{ABSTRACT}

The response of Obama's Administration to Occupy Wall Street movement is an instance of institutional rhetoric of fear. For this reason, this paper offers a rhetorical approach of some of texts used by Occupy Wall Street and the interaction of this movement and American Government.
\end{abstract}

KEYWORDS: UNITES STATES * OCCUPY WALL STREET * RHETORIC * FEAR * POLITICS

Escuela de Filología, Lingüística y Literatura de la Universidad de Costa Rica (UCR). hcamposv@yahoo.es 


\section{ANTECEDENTES}

¿Alguna vez hemos temido expresar nuestras ideas? ¿Cuántas veces por miedo callamos ante una injusticia? En efecto, vivimos en ambientes profundamente marcados por el temor.

Aristóteles, en el libro segundo de la $R e$ tórica, ubica el miedo en el campo de las pasiones. Sobre este afirma que su base se encuentra en que: “... el miedo es un cierto pesar o turbación, nacidos de la imagen de que es inminente un mal destructivo o penoso" (1999: 334). Ahora bien, prosigue el pensador de Estagira: “... no todos los males producen miedo sea, por ejemplo, el ser injusto o el ser torpe, sino los que tienen capacidad de acarrear grandes penalidades o desastres, $y$ ello además si no aparecen lejanos, sino próximos, de manera que estén a punto de ocurrir" (ibid: 334-335). El apartado dedicado a este tema concluye en los siguientes términos:

Por lo tanto, conviene poner a los <oyentes $>$, cuando lo mejor sea que ellos sientan miedo, en la disposición de que puede sobrevenirles un mal (pues también lo sufrieron otros superiores a ellos) $y$ mostrarles que gentes de su misma condición lo sufren o lo han sufrido, $y$, además, de parte de personas de las que no cabría pensarlo y por cosas y en momentos que no se podrían esperar (ibid: 338).

El año 2011 fue un periodo de insurgencias, es decir, manifestaciones que implicaron una pérdida del temor: desde Magreb, Yemen y Siria hasta España, Chile, Israel y Nueva York. Algunas de estas fueron objeto de metáforas meteorológicas: la primavera árabe, el verano europeo y el otoño estadounidense, son los ejemplos más destacados.

Estos movimientos rompieron los modelos políticos a los que están acostumbrados la mayor parte de los analistas, ya que no se trata de partidos políticos, no cuentan con una organización individual que los identifique, carecen de un vocero o representante oficial $y$ no ofrecen un manifiesto programático o de intenciones, entre otros rasgos destacados.
Los movimientos sociales y los partidos políticos son fenómenos distintos que se mueven en diferentes niveles de acción. Los partidos políticos lo hacen en el nivel de la representación de intereses mientras que los movimientos sociales contribuyen a redefinir las coordenadas culturales y políticas en las que tiene lugar la representación de intereses (Romanos, 2012: 2).

Por ejemplo, del 15M, movimiento de origen español, Eduardo Romanos indica:

...es un fenómeno más amplio y complejo, articulado en forma de red por la que circulan densos flujos informales de interacción entre actores que comparten una identidad colectiva (se sienten parte de una comunidad de "indignados") y mantienen una relación de conflicto con quienes, a su juicio, son los responsables del problema social que denuncian en sus protestas (2012: 2).

En occidente, estos grupos enarbolan una crítica al liberalismo económico en busca de un concepto que parecía haber caído en desuso: el bien común.

Para Benjamín Arditi, se trata en general, de insurgencias que desafían el statu quo:

Las insurgencias no son prácticas de política cotidiana o ejercicios de elaboración de políticas públicas. Son actos colectivos en los que la gente dice "ibasta!" y se niega a continuar como antes. Ellas son el plan en tres sentidos: su mera existencia las convierte en operadores de la diferencia en relación con lo dado, son performativos políticos - los participantes comienzan a experimentar aquello por lo cual luchan y funcionan como mediadores evanescentes o portales hacia posibilidades que pueden o no materializarse. Estos tres puntos se retoman en una discusión acerca del remanente material de dos insurgencias, la primavera árabe y las movilizaciones estudiantiles en Chile (2012: 1). 
Este mismo estudioso, cita a Immanuel Wallerstein, quien considera que Occupy Wall Street es: "el suceso político más importante en los Estados Unidos desde los levantamientos de 1968, de los que es descendiente directo o continuación". Concluye diciendo que habrá triunfado y dejado un legado incluso si se desintegra debido al cansancio o la represión (2011, citado en Arditi, 2012: 12). Este tipo de fenómeno, ante la ausencia de una agenda política específica, grosso modo:

Expresaban el hartazgo con la impunidad de los poderosos y con la farsa de una justicia social inexistente. Sus banderas incluían los derechos humanos y la democracia, la educación gratuita y laica, la vivienda asequible, el responsabilizar a las empresas financieras por su papel en la crisis, la obscenidad de una distribución tan desigual del ingreso, la falta de empleos $y$ perspectivas de futuro para la mayoría de la gente y la insatisfacción con los políticos corruptos e incompetentes. Su enojo se plasmaba en inscripciones poéticas como "Si no nos dejan soñar, no los dejaremos dormir", "Sólo porque no lo ves no significa que no esté ocurriendo" y "Nadie puede predecir el momento de la revolución", así como en consignas como "Somos el 99\%" y "Wall Street es nuestra calle" que servían para generar identidad (Arditi, 2012: 3).

Para los estudiosos, pese a su poca o falta de adecuación a los objetos de estudio tradicional, se presentan muy productivos, pues:

El remanente material de las insurgencias también se observa en los artefactos culturales que dejan: canciones, grafitis, manifiestos, panfletos, fotografías, películas, blogs, sitios de internet $y$ una serie de testimonios en los medios sociales como Twitter y Facebook. También está el predecible torrente de congresos, talleres, publicaciones (incluida ésta), entrevistas, análisis de los medios, evaluaciones por parte de los $y$ las activistas y conversaciones cotidianas que intentan comprender la experiencia de estas insurgencias tiempo después de que ya pasaron (Arditi, 2012: 17).

Cronológicamente, uno de los antecedentes directos con mayor influencia para Occupy Wall Street fue el movimiento de los indignados, en particular, el conocido como 15M (derivado de la fecha 15 de mayo de 2011, día en que se ocupó Madrid). Sus raíces pueden rastrearse hasta la década de 1960, cuando surgieron los movimientos anarquistas, ecológicos, etc., muchos de los cuales, posteriormente, se manifestaron como partidos políticos. Se trata de un movimiento social, asociado con prácticas de la democracia deliberativa orientadas a la consecución de una justicia social global.

Uno de sus instrumentos consiste en la "toma”, la "ocupación" de espacios públicos, así en España, Egipto, Baharein y Nueva York, como se ha señalado supra. Una de las características que destaca al 15M respecto de otros movimientos sociales es haber trasladado la democracia participativa al espacio público tomado.

\section{VIRTUDES RETÓRICAS DE OCCUPY WALL STREET}

Occupy Wall Street (ows) inició el 17 de septiembre de 2011, como señal de protesta y presión en el Zuccotti Park.

Desde un punto de vista de práctica social, su existencia rompe con la normalidad y la normatividad, experiencia que a nivel incidental se experimenta cotidianamente con las protestas e interrupciones del tránsito. Empero, su permanencia en el tiempo constituye una severa infracción a las prácticas habituales. En los llamados "Estados de Derecho", tales tipos de actividades están prohibidas en virtud de principios como los de libertad de tránsito, seguridad e incluso, salud pública.

La existencia de normas prohibitivas, acompañadas de sanciones de orden pecuniario $y$ prisión (conocidas como las manifestaciones de la "prevención especial y general" del derecho penal), suelen ser los mecanismos de contención a los que el aparato estatal apela para mantener el "orden público". No obstante, estos 
recursos intimidatorios y disuasorios pierden su eficacia ante estos eventos.

La amenaza general al patrimonio y la libertad de los manifestantes no fue suficiente. Esta ruptura parece responder primeramente, al exemplum ofrecido por el 15M (el movimiento de los "indignados" en España), en segundo término, al carácter multitudinario del evento, no necesariamente grupal, lo cual constituye una situación ajena a los mecanismos de control ordinarios y en tercer lugar — quizá el más significativo desde el punto de vista retóricoa la desaparición del temor.

Efectivamente, puede partirse de la hipótesis de que la mayor parte de los manifestantes, al haber perdido todo lo que era significativo para ellos (trabajo y techo), no tenían en aquel momento nada más que perder, por lo que pudieron arriesgarlo todo.

A partir de este momento, el receptor de la "retórica del temor" institucional desapareció y se constituyó en "orador" merced, no tanto a un examen racional del statu quo, sino a un contexto pasional:

Porque las pasiones son, ciertamente, las causantes de que los hombres se hagan volubles $y$ cambien en lo relativo a sus juicios, en cuanto que de ellas se siguen pesar y placer. Así son, por ejemplo, la ira, la compasión, el temor y otras más de naturaleza semejante y sus contrarias (Aristóteles, 1999: 310).

En este sentido, la propia identificación del 15M español brindada por los medios mediante el apelativo "los indignados", es elocuente. La toma de consciencia de esta estrategia "institucional" quizá ha motivado precisamente, su rechazo por el propio movimiento, el cual ha preferido identificarse mediante una referencia a la fecha de inicio de la protesta. Ciertamente, "indignados" alude más a una situación pasional, visceral si se quiere, que a un cuestionamiento racional, lo cual implica de alguna manera una forma de descalificación.

Occupy Wall Street ha sufrido vicisitudes semejantes, ya que la primera forma de ataque ha sido la descalificación y la agresión.
La descalificación ha aprovechado la naturaleza propia del movimiento, el cual, no solo en su dinámica (ocupar un espacio público por un tiempo prolongado) sino que en su constitución representa una "anormalidad".

Por lo general, el ser humano tiende a conocer los fenómenos nuevos a través de lo conocido. Respecto a Occupy Wall Street, el fenómeno conocido son los partidos políticos, incluso, en ese proceso se le ha comparado con el Tea Party, un movimiento que, contrario a Occupy Wall Street, ha tenido incidencia y proyección políticas. No obstante, Occupy Wall Street no es un partido político, ni por el momento ha pretendido serlo.

A pesar de que los movimientos sociales son muy antiguos, su estudio retórico es complejo, ya que se trata de subsumirlos en el modelo de los partidos políticos. La razón es sencilla: es más fácil estudiar y examinar un partido político (con su estructura, organización, proyecto político, representantes e incluso, patrimonio inmobiliario) que fenómenos de este tipo.

Los estudios retóricos no escapan a este tipo de problemas, pues la tendencia es aplicar principios generales de retórica clásica que pretenden identificar a un orador, un texto y una audiencia. Occupy Wall Street presenta un serio problema de opacidad retórica en este sentido: ¿quién es el orador?, ¿cuál es el texto? y ¿cuál es la audiencia?

De estas tres preguntas, quizá la última sea la que pueda responderse con mayor facilidad: el público y el gobierno norteamericanos.

Sobre el texto, la ambigüedad es manifiesta $y$ en algunos casos, ha sido aprovechada por la crítica institucional. Por ejemplo, acciones comunes como movimientos rítmicos de individuos o de masas, bailar, pintarse los rostros con tiza, caminar, repetir una misma palabra con distintas variaciones... ¿qué clase de texto son? Este tipo de comportamientos, identificados como Urban Play, son desconcertantes para el crítico retórico. Algunos manifestantes se expresan así respecto a tal práctica:

... nosotros seguimos la intensidad afectiva de nuestra dinámica juntos, repitiendo movimientos que ofrecen un alto 
nivel de intensidad y transformándolos cuando un nuevo movimiento captura nuestro interés y muestra intensidad (Landers, 2012: 5) ${ }^{1}$.

La descalificación era el corolario inminente tal y como se aprecia en algunas críticas; por ejemplo:

Joanna Weiss, del Boston Globe, ha dicho al respecto: "Es difícil tomar en serio una protesta cuanto se ve como un circo..." (2011: 2). En el New York Times, Gina Bellafante expresó su escepticismo acerca de la efectividad de "ventear la queja social a modo de carnaval", cuando la magnitud de los problemas que subyacen al capitalismo "no son fácilmente corregibles por medio del teatro callejero" (2011: 1). Reconocidos activistas, tanto dentro como fuera del movimiento, han ofrecido su crítica contra nuestra jovialidad en forma de consejo, pidiéndonos que dejemos de jugar alrededor $y$ hagamos algunas demandas (Brookings Institution, 2011; Hoffman, 2011 y Moore, 2011). ${ }^{2}$

Por su parte, también se ha expresado:

Bloomberg, el multimillonario alcalde de Nueva York, un claro representante de alto nivel tanto del $1 \%$ que controla al Estado como del Estado que es controlado por el $1 \%$, ha justificado su

$1 \quad(\ldots)$ we track the affective intensity of our play together, repeating movements that present a high level of intensity and transforming them when a new movement captures our interest and shows intensity.

2 Said Joanna Weiss of the Boston Globe, "It's hard to take a protest fully seriously when it looks like a circus...." $(2011,2)$. Ginia Bellafante in the New York Times expressed skepticism about the effectiveness of "air[ing] societal grievances as carnival," when the magnitude of problems under capitalism are "not easily extinguishable by street theater" $(2011,1)$. Established activists, both inside and outside of the movement, offered their criticism of our playfulness in the form of advice, urging us to stop playing around and make some demands. violencia contra la Ocupación, señalando que el propósito del juego, del disfrute en particular $y$ de la intensidad afectiva en general, no es relevante para alcanzar un cambio social. "Es divertido y es catártico... es, no sé si es divertido ir y culpar gente" (Taylor, 2011: 1 y Landers, 2012: 3$)^{3}$.

Aunado a lo anterior, surge la pregunta institucional "¿qué queréis?”, a la cual es difícil responder por un movimiento sin representantes ni estructuras preconstituidas.

No se puede hablar de un texto sino de múltiples textos, ejemplificados particularmente a través de uso del slogan, entre los que cabe citar los siguientes:

"Nosotros somos el 99 por ciento".

"Somos demasiado grandes para fallar".

"Necesidades humanas, no avaricia corporativa".

"Generación de la revolución... tener deudas es esclavitud".

"Perdieron mi trabajo, encontraron una ocupación".

"La libertad de empresa no es una licencia para cazar".

“Trabajaremos por dinero".

3 Billionaire New York City Mayor Bloomberg, a high profile representative of both the $1 \%$ that controls the state and the state that is controlled by the $1 \%$, has justified his violence against the Occupation by implying that the goal of play, enjoyment in particular and affective intensity in general, is not relevant to achieving social change. "It's fun and it's cathartic - it's, I don't know, it's entertaining to go and to blame people". 
"Fuera de nosotros".

"USA=Asociación de accionistas unidos".

"La gente por encima de los beneficios".

"Salven el sueño americano".

"De "esparciendo la riqueza" a "esparciendo la miseria"”.

"Un día los pobres no tendrán nada más que comer que gente rica".

"Los bancos fueron rescatados, nosotros fuimos vendidos".

"Wall Street debería comprar acciones, no políticos".

"Saquen el dinero de la política".

"OccuPY: Superar la corrupción corporativa mediante la unión de nuestro pueblo, sî".

"Yo no soy su ATM".

"Es la segunda vez que peleo por mi país, la primera vez conocía a mi enemigo".

"Tiremos el amiguismo congresista no hace nada".

"Mi nombre es Inigo América. Ustedes asesinaron mis libertades. Prepárense para el cambio".
"Si el voto hiciera una diferencia, yo ya habría hecho la diferencia".

"Nosotros somos las cansadas masas hacinadas de pobres".

"No podemos resolver problemas con la misma forma de pensamiento que usamos para crearlos" (Albert Einstein) ${ }^{4}$.

Se trata de afirmaciones a las que es fácil asentir, lo cual desde un punto de vista argumentativo es de suma importancia.

Algunos de estos son máximas en sentido aristotélico, es decir, se trata de: “... una aseveración universal; pero de lo que aquellos se complacen es de que se diga universalmente lo que ya a ellos les había acontecido comprender antes en particular" (Aristóteles, 1999: 415). Sin embargo, es fácil descubrir cierto tipo de humor en su composición:

Algunos experimentos en psicología social y los estudios sobre la comunicación han mostrado cómo el humor favorece la recepción de los mensajes, al atraer el interés de la audiencia hacia al acto de la comunicación. El humor a

$4 \quad$ "We are the 99 percent", "We are Too Big to Fail", "Human Need, Not Corporate Greed", "Revolution Generation... Debt is Slavery", "Lost My Job, Found an Occupation", "Free Enterprise is Not a Hunting License", "Will Work for Money", "Out OURCED", "USA=United Shareholder Association", "People Over Profits", "Save the American Dream", "From "Spreading the Wealth" to "Spreading the Misery", "One Day the Poor Will Have Nothing to Eat but the Rich", "Banks Got Bailed Out, We Got Sold Out", "Wall Street Should Buy Stocks, Not Politicians", "Get Money Out of Politics", "Occupy: Overcome Corporate Corruption by Uniting Our People, Yes", "I am Not Your ATM", " $2^{\text {nd }}$ Time I've Fought for My Country, $1^{\text {st }}$ Time I've Know My Enemy", "Dump the Crony Do Nothing Congress", "My Name is Inigo America. You Killed My Freedoms. Prepare to Change", "If Voting Made a Difference, It Would Already Have Made a Difference", "We are the Tired Poor Huddled Masses", "We Can't Solve Problems by Using the Same Kind of Thinking We Used When We Created Them" Albert Einstein. 
menudo resulta en una mejor respuesta positiva $y$, además, en alguna medida, otorga credibilidad a la imagen del emisor, con lo que facilita que los mensajes posteriores sean recibidos de una manera más positiva (Gruner, 1970; Markiewicz, 1974; Wanzer, Booth-Butterfield y Booth-Butterfield, 1996, citados en Romanos, 2012: 10) 5 .

De esta manera, el humor ofrece ciertas propiedades retóricas, al generar no solo un pathos específico, sino incidir en el logos y en el ethos.

En muchos de estos slogans se aprecia el recurso a la denominada culture jamming, la cual puede ser explicada en los siguientes términos:

Esto se refiere a una estrategia simbólica por medio de la cual los activistas en contra de las corporaciones hacen uso de diversas técnicas estéticas (por ejemplo: la apropiación, el collage, la inversión irónica, la yuxtaposición) para cambiar las recomendaciones del discurso corporativo original mediante la alteración visual de los símbolos de las corporaciones (logos, slogans, etc.) y así les dan un nuevo significado (al respecto, puede consultarse Wettergren, 2009; Van Laer y Van Aelst, 2010, citado en Romanos, 2012: 12) .

$5 \quad$ Various experiments in social psychology and communication studies have shown how humor favors the reception of messages, attracting the interest of the audience into the act of communication. Humor often results in a more positive response and, indeed in some way gives credibility to the image of the communicator, thus facilitating future messages being received in a more positive way (Gruner 1970; Markiewicz 1974; Wanzer, Booth-Butterfield and Booth-Butterfield, 1996).

This refers to a symbolic strategy by way of which anti-corporate activists make use of diverse artistic techniques (e.g., appropriation, collage, ironic inversion, juxtaposition) to change the original discourse of corporate advertising by altering corporate symbols (logos, slogans, etc.) visually and thus giving them a new meaning (see Wettergren, 2009; Van Laer and Van Aelst, 2010).
En general, los slogans participan de las tres cualidades (virtutes) de la narratio: la brevedad (brevitas), la claridad (claritas/perspicuitas) y la verosimilitud (verisimilis/probabilis) fortaleciendo sobremanera el proceso comunicativo (sobre el tema puede consultarse: Mortara, 2000:76).

A su vez, aparecen asociados a los procesos de comunión descritos por Perelman y Olbrechts-Tyteca. Se trata de uno de los efectos discursivos más importantes para estos autores, al punto de constituir una de las tres principales figuras estudiadas por ellos (las otras dos son las figuras de elección y las de presencia). Las de comunión, tal y como su nombre sugiere:

... son aquellas con las que por medio de procedimientos literarios, el orador se esfuerza por crear o confirmar la comunión con el auditorio. A menudo, esta comunión se obtiene gracias a las referencias a una cultura, una tradición o un pasado comunes (1989: 282, §42).

A la comunión se asocian figuras retóricas como la cita, la alusión, el apóstrofe, la interrogación retórica, entre otras.

De este grupo de slogans, los siguientes destacan por estar más asociados a las figuras de comunión:

$$
\text { “iNosotros somos el } 99 \text { por ciento!” }
$$

“Somos demasiado grandes para fallar!”

\section{“iSalven el sueño americano!”}

Por otra parte, las de elección procuran transmitir información relevante al oyente con fines argumentativos tendientes, principalmente, a plantear el punto de vista del emisor. Figuras retóricas como la interpretación, la definición, la perífrasis y la prolepsis suelen aprovechar este valor. Con el valor de la elección destacan:

“¡Nosotros somos el 99 por ciento!”.

"Generación de la revolución... tener deudas es esclavitud". 
"La libertad de empresa no es una licencia para cazar".

"Mi nombre es Inigo América. Ustedes mataron mis libertades, prepárense para el cambio".

"Nosotros somos las cansadas masas hacinadas de pobres".

"No podemos resolver los problemas usando la misma forma de pensar que usamos para crearlos" (Albert Einstein).

En las figuras de presencia, cuya función es traer a la mente del auditorio el objeto del discurso, destacan la repetición, la anáfora y la sinonimia. A este grupo de figuras están asociadas expresiones como las siguientes:

"Los bancos fueron rescatados, nosotros fuimos vendidos".

"Si el voto hiciera una diferencia, yo ya habría hecho la diferencia".

"Perdieron mi trabajo, encontraron una ocupación”.

"Fuera de nosotros".

"USA=Asociación de accionistas unidos".

"Tiremos el amiguismo congresista no hace nada".

"Un día los pobres no tendrán nada más que comer que gente rica".

"OCCUPY: Superar la corrupción corporativa mediante la unión de nuestro pueblo, sî".
"Sí, no soy tu ATM".

"Es la segunda vez que peleo por mi país, la primera vez conocía a mi enemigo".

De lo anterior, puede colegirse que pese a la debilidad que, en principio, podría representar la ausencia de una estructura definida de Occupy Wall Street, en el plano retórico presenta fortalezas significativas.

\section{LA RESPUESTA RETÓRICA INSTITUCIONAL: LA RETÓRICA DEL MIEDO}

Ante estas propiedades, la respuesta institucional fue sistemática. El 5 de octubre de 2011, el Secretario de Prensa de la Casa Blanca, fue interrogado en estos términos:

Una última cosa. Ha habido algunas revueltas en Grecia hoy y el Alcalde Bloomberg recientemente sugirió que, debido al alto desempleo, podría haber revueltas en las calles de los Estados Unidos. Ahora mismo no tenemos revueltas, pero existe el movimiento Occupy Wall Street que está en marcha. ¿Cuál es la visión de la Casa Blanca acerca de Occupy Wall Street y qué piensa usted sobre las revueltas en Grecia y sobre si algo semejante podría ocurrir o no aquí? (Office of the Press Secretary of the White House, 5 de octubre de 2011) 7 .

La respuesta inició de la siguiente manera:

Bien, haré a un lado la comparación con Grecia. El hecho es que afuera hay americanos que están comprensiblemente frustrados con la economía, con las dificultades para encontrar trabajo o

7 Last thing. There were some riots in Greece today, and Mayor Bloomberg recently suggested that because of high unemployment there could be riots in the streets of the United States. And right now we don't have riots but there's the Occupy Wall Street movement that's going on. What's the White House view about Occupy Wall Street, and what do you think about the riots in Greece and whether or not something like that could happen here? 
mantenerse en uno. Yo creo que esto es exactamente por lo que el Presidente está - a pesar del menosprecio de Billhaciendo una gira a lo largo del país en la que se expone la urgente necesidad de que el Congreso apruebe la Ley sobre el trabajo americano.

Yo creo que es comprensible que haya frustración. Debo recordarle que este Presidente ha peleado - aunque no siempre sea agradable - para asegurar que se aprueben amplias protecciones para el consumidor en la Ley de reforma financiera que fue rechazada por el Congreso, cuyos miembros - digo, del Congreso, los Republicanos en el CongresoRepublicanos que ahora quieren revocarla.

¿Por qué? En parte porque millones $y$ millones de dólares han sido gastados en lobby contra esta ley por empresas que no la quieren. Pero - vea, caímos en un terrible desastre debido en parte a las acciones que se tomaron en Wall Street. Dos cosas son ciertas acerca de las acciones tomadas por este Presidente a raíz de que asumió el cargo durante la peor recesión desde la Gran Depresión- una contracción catastrófica en la economía, una catastrófica pérdida de empleos ${ }^{8}$.

8 Well, I'll leave aside the comparison to Greece. The fact is that there are Americans out there who are understandably frustrated with the economy, with their difficulty in finding a job or holding onto a job. I mean, this is exactly why the President is - despite Bill's contempt- barnstorming around the country arguing for the urgent need for Congress to act on the American Jobs Act.

So I think that it's understandable that there's frustration. And need I remind you that this President fought — and it wasn't always prettyto make sure that we passed sweeping consumer protections in the Financial Reform Act that was opposed by Congress, and that which members - I mean, Congress, by Republicans in CongressRepublicans now who want to repeal it.

Why? I mean, in part because millions and millions of dollars are being spent to lobby against it by the industries who don't like it. But the -look, we
En el contexto mundial de los disturbios vividos en Grecia, la reacción del Secretario Carney es estratégica: hace a un lado la comparación. Inmediatamente, se asocia al movimiento con cólera - anger, es la forma como la misma prensa norteamericana lo ha designado (Office of the Press Secretary of the White House, 17 de octubre de 2011). Esta expresión cuenta con un antecedente en el movimiento de los indignados en España, donde:

Los medios han destacado el elemento de la indignación y esto es lo que ha hecho que el movimiento sea conocido internacionalmente como los indignados. Esta etiqueta hace referencia al libro ¡Indignáos!, por Stéphane Hessel, que algunos identifican como la principal fuente de inspiración para quienes protestan en España. Los activistas, sin embargo, prefieren autodenominarse como el Movimiento $15 \mathrm{M}$, en memoria a la fecha en que principió el movimiento, el 15 de mayo del 2011. La indignación es, sin lugar a dudas, la emoción central de este movimiento español (Romanos, 2012: 1) ${ }^{9}$.

Seguidamente, identifica el origen y expresión del movimiento: hay frustración, la acepta, no como simple concesión retórica, sino que la aprovecha para fundamentar la acción del Presidente Obama en su plan de reformas legislativas.

got into an awful mess because of in part some of the actions that were taken by Wall Street. And two things are true about the actions this President took in the wake of that, when he took office during the worst recession since the Great Depression- a catastrophic contraction in the economy, catastrophic job loss.

9 The media have stressed the element of indignation and this is how the movement has become known internationally, the indignados. The label makes reference to the book Indignez-vous! by Stéphane Hessel, which some identify as a main source of inspiration for the Spanish protests. The activists, however, prefer to call themselves the $15 \mathrm{M}$ movement, in honor of the date that was the starting gun for the movement, May 15th, 2011. Indignation is, without a doubt, a central emotion in the Spanish movement. 
Si bien es cierto, se trata de una pasión y por lo tanto, un impulso irracional, se vinculó con aspectos que bien pueden considerarse racionales, tales como la debacle de la economía, la necesidad de mejorar la generación de empleo y la necesidad de mejorar el crecimiento, por lo que no se descalificó al movimiento.

Adicionalmente, los medios y en especial el gobierno norteamericano, identificaron a los manifestantes con un estrato específico de población, la clase media norteamericana. Como respuesta a esta acción, la fraseología generada destaca "we are the 99 percent of Americans", en clara alusión al gran contingente de la población que no es multimillonaria.

Ahora bien, la administración Obama desplegó una fuerte retórica del miedo contra Occupy Wall Street, materializada a través de acciones de distinto grado. Esto llevó al movimiento a responder de muy diversas formas. Por ejemplo, contra una acción sutil como el prohibir el uso de amplificadores:

Los activistas inventaron el micrófono humano para darle la vuelta a la decisión del Departamento de Policía de Nueva York de prohibir el uso de megáfonos y amplificadores en actos públicos a menos que éstos contaran con un permiso que no tenían intención de concederles. Se trata de una solución anacrónica y decididamente low tech surgida de jóvenes que se sienten más a sus anchas con el uso de las nuevas tecnologías de la información. El familiar "¡Mic check!” [prueba de sonido] podía oírse en los actos de los ocupas cuando un orador u oradora subía al podio sin micrófono en la mano o la solapa. La frase preparaba a la asamblea para una manera inusual de amplificar el sonido: la gente repetía a coro lo que decía la persona que hablaba para que quienes estaban lejos también pudieran escuchar (Arditi, 2012: 17).

También como parte del genio inventivo de esta organización: "Las asambleas de Ocupa Wall Street adaptaron un lenguaje de señas con las manos para expresar acuerdo, desacuerdo, moción de orden o el bloqueo de propuestas" (ibid).

De esta manera, se rompió lo que los psicólogos llaman "la impotencia aprendida" (learned helplessness):

... no podemos olvidar el terror generado por el hostigamiento cotidiano, la detención arbitraria y la tortura. El terror busca infundir una desconfianza paranoica hacia los demás y la creencia de que toda resistencia es inútil porque nada escapa a la mirada y los oídos de la policía y de su red de informantes (Arditi, 2012: 13).

Siempre en el plano retórico, la lucha asumió otras vertientes. En noviembre de 2011, durante la reunión de la Republican Governors Association (Asociación de Gobernadores Republicanos), celebrada en Florida, con el propósito de definir políticas y estrategias de trabajo, Frank Luntz, estratega republicano y uno de los más destacados expertos de comunicación política, estableció diez recomendaciones básicas para los republicanos, todas de carácter retórico:

1) No digan capitalismo, digan libre mercado o economía libre, ya que el concepto de capitalismo, aunque se prefiere frente al socialismo, se asocia con inmoralidad.

2) No digan tomar dinero del pueblo, sino gravar al rico.

3) No digan clase media, digan los esforzados trabajadores que pagan sus impuestos.

4) No digan trabajos, digan carreras.

5) No digan el gobierno gasta, digan el gobierno desperdicia.

6) No digan compromiso, digan cooperación.

7) Digan a los occupiers, los entendemos.

8) Digan propietarios de pequeños negocios o creadores de fuentes de empleo, en lugar de innovadores o empresarios.

9) Nunca digan sacrificarse, digan todos estamos juntos en esto.

10) Siempre culpen a Washington (Moody, 2011. La traducción es del autor). 
Por último, Luntz recomendó, dada la proximidad de la época navideña en ese entonces, no emplear el término bonus, en cambio, sugirió la expresión pago por su cumplimiento.

Otras acciones, en cambio, fueron abiertamente represivas. Por ejemplo: “... los Ocupantes de Wall Street fueron golpeados, rociados con gas pimienta $y$ arrestados por la policía, son criticados por activistas y por la prensa debido a que juegan demasiado alrededor cuando deberían estar formulando sus demandas"(Landers, 2012: 5) ${ }^{10}$.

A fines de enero de 2012, cerca de 400 manifestantes del movimiento Occupy Wall Street fueron arrestados en Oakland, mientras el Servicio Nacional de Parques planeaba retirar a otro grupo de manifestantes de un sitio del Dc. Interrogado al respecto, el Secretario de Prensa, Jay Carney, expresó:

Nuestra posición ha sido y continúa siendo que necesitamos encontrar un balance entre la Primera Enmienda $y$ la libertad de expresión, el derecho de hablar libremente, comprometidos con la seguridad y la salud públicas. Comprendemos que si - el esfuerzo de la autoridad local, así como, en este caso, el Servicio Nacional de Parques y la Policía de Parques de los Estados Unidos están ponderando estos aspectos al tomar estas decisiones, eso es apropiado (Office of the Press Secretary of the White House, 30 de enero de 2012) ${ }^{11}$.

$10 \quad(\ldots)$ Wall Street Occupiers were beaten, pepper sprayed, and arrested by police, and criticized by activists and the media for playing around too much when they should have been making demands.

11 And our position has been and continues to be that we need to balance First Amendment concerns of the right to demonstrate, the right to speak freely, with public safety concerns and public health concerns. And we understand that whether - that local law enforcement, as well as, in this case, the National Park Service and U.S. Park Police, are weighing those considerations when they make these decisions, and that's appropriate
La respuesta apela a la metáfora de la balanza (asociada a las ideas de justicia y equilibrio): en uno de sus brazos se encuentran el derecho de manifestación y expresión; en el otro, la preocupación por la seguridad y salud públicas.

Los arrestos nunca fueron extraños contra este movimiento. Así, luego de una serie de detenciones a mediados de noviembre de 2011, se le preguntó al Secretario de Prensa, Jay Carney, sobre la posición del Presidente Obama, a lo que respondió:

Él es consciente de eso, obviamente, a partir de los reportes. Y nuestra posición, así como la posición del Presidente, es que obviamente todo municipio tiene que tomar sus propias decisiones acerca de cómo manejar estos temas. Nosotros esperaríamos y querríamos, tal y como estas decisiones han sido tomadas, que esto muestre un equilibrio entre una larga tradición de libertad de reunión y libertad de expresión en esta nación, obviamente, denunciando y protestando, y además la importantísima necesidad de mantener la ley y el orden, la salud y estándares de seguridad, que han sido obvias preocupaciones en este caso (Office of the Press Secretary of the White House, 15 de noviembre de 2011) ${ }^{12}$.

La primera respuesta es una definición: los organismos municipales son las entidades competentes para tomar decisiones sobre cómo manejar estos asuntos. La segunda parte de su respuesta involucra nuevamente la imagen de la balanza, al proponer que se ponderen diversos factores: por un lado, las libertades

12 He's aware of it, obviously, from the reports. And our position and the President's position is that obviously every municipality has to make its own decisions about how to handle these issues, and we would hope and want, as these decisions are made, that it balances between a long tradition of freedom of assembly and freedom of speech in this country and obviously of demonstrating and protesting, and also the very important need to maintain law and order and health and safety standards, which was obviously a concern in this case. 
de reunión, de expresión, de manifestación y de protesta; por el otro, el mantenimiento de la ley y el orden, así como, los estándares de salud y seguridad.

La propia administración de Obama asumió el fenómeno retóricamente en su propio provecho. Así, a manera de ilustración, al conmemorarse el primer aniversario de este "movimiento" (así ha sido llamado por la prensa y el gobierno norteamericano), el propio Secretario de Prensa, Josh Earnest, valora como uno de los resultados positivos de este fenómeno, el aumento del debate - un mayor incremento del debate en este país ${ }^{13}$, o conversación- al menos de la conversación en este país ${ }^{14}$ (Office of the Press Secretary of the White House, 17 de setiembre de 2012), sobre la clase media norteamericana.

En dicha entrevista, Josh Earnest emplea en dos ocasiones la misma expresión: "la peor recesión desde la Gran Depresión"15 (Office of the Press Secretary of the White House, 17 de setiembre de 2012). En igual número de ocasiones, Jay Carney apeló a esta idea en una entrevista realizada el 17 de octubre de 2011: el mismo presidente Obama reconoce la realidad de esta comparación.

En el plano legislativo, en algunas ocasiones sirvió de palanca para promover la aprobación de dos proyectos de ley, a saber: La Ley de Reforma de Wall Street ${ }^{16}$ y la Ley sobre el Trabajo Americano ${ }^{17}$, las cuales fueron presentadas como la cura contra el mal que suscitó la ocupación.

El Presidente Obama pocas veces ha hecho referencia al movimiento. Una de las pocas ocasiones en que lo ha mencionado, fue al contestar una serie de preguntas de esta manera:

Obviamente, yo he escuchado sobre eso. Lo he visto por televisión. Yo creo que eso expresa las frustraciones que el pueblo

\footnotetext{
13 "further elevation of the debate in this country".

14 "at least the conversation in this country".

15 "the worst economic downturn since the Great Depression".

16 Wall Street Reform Act.

17 American Jobs Act.
}

americano siente ante la mayor crisis financiera que hemos tenido desde la Gran Depresión, los amplios daños colaterales que se han producido a lo largo de toda la nación, a lo largo de Wall Street, y todavía vemos algunos de los mismos tipos que actuaron irresponsablemente tratando de luchar contra los esfuerzos por acabar con las prácticas abusivas que nos llevaron a este problema en primer lugar (Office of the Press Secretary of the White House, 17 de octubre de 2011: 7) ${ }^{18}$.

El distanciamiento entre la persona del Primer Mandatario de los Estados Unidos y Occupy Wall Street es manifiesto: es de oídas $y$ por la televisión que se "ha enterado de él", la descalificación simbólica es patente. De inmediato, luego de asociarlo con el sentimiento de frustración, lo asume como arma contra los opositores a su plan de reforma, con lo que el centro de su discurso siempre ha sido su administración.

Ahora bien, no es cierto que la crítica que representa Occupy Wall Street sea solo frustración ante la mayor crisis financiera desde la Gran Depresión. Este ha sido el detonante que parece haber abierto los ojos de muchos, pero la crítica no ha sido esta. A través de su discurso, Obama y su gabinete construyen una ecuación:

Ocupantes=frustración $=$ la más grande crisis financiera desde la Gran Depresión

Sin embargo, es posible construir ecuaciones como:

Ocupantes $=$ crítica $=$ sistema económico

18 Obviously I've heard of it. I've seen it on television. I think it expresses the frustrations that the American people feel that we had the biggest financial crisis since the Great Depression, huge collateral damage all throughout the country, all across Main Street, and yet you're still seeing some of the same folks who acted irresponsibly trying to fight efforts to crack down on abusive practices that got us into this problem in the first place. 

al afirmar:

El Presidente Obama reconoce esta tesis

Así, en efecto, creo que la gente está frustrada, y los manifestantes están dando una voz a una base más amplia de frustración acerca de cómo nuestro sistema financiero trabaja. Ahora, recuerde lo que yo he dicho antes y que seguiré repitiendo: tenemos que tener un sector financiero fuerte $y$ efectivo para que crezcamos (ibid) $)^{19}$.

Ante estas otras posibilidades, Obama responde categóricamente y sin ambages que cree en la necesidad de un sector financiero efectivo para alcanzar el crecimiento de la Nación.

Pensar en la Gran Depresión es mirar al pasado, un evento financiero que afectó, como el presente, al mundo entero, pero que fue superado: se trata de una analogía política con muy sanos efectos. Nótese que la presente no se configura como "la peor crisis financiera", ni tampoco se establece una comparación directa con la Gran Depresión, la cual podría arrojar resultados comprometedores que debilitarían la analogía al posiblemente mostrar que se está ante una crisis peor que la de la Gran Depresión (conclusión que pondría en tela de juicio la capacidad de los Estados Unidos de superarla).

Nada más estratégico, en un momento de crítica, que volver la mirada hacia al pasado. Así, al compararse este movimiento con el Tea Party, Obama contestó:

Lo que pienso es que el pueblo americano comprende que no todos han estado siguiendo las reglas, que Wall Street es un ejemplo de esto, que la gente que trabaja duro cada día se levanta, va a su trabajo, es leal a su compañía, y que esto solía ser la esencia del Sueño Americano. Así es como se avanza, a la manera antigua. En estos días, gran cantidad de

19 So, yes, I think people are frustrated, and the protestors are giving voice to a more broad-based frustration about how our financial system works. Now, keep in mind I have said before and I will continue to repeat, we have to have a strong, effective financial sector in order for us to grow. personas que están haciendo lo correcto no son recompensados, mientras que gran cantidad de personas que no están haciendo lo correcto sí lo son.

Y esto es lo que se va a expresar políticamente en el 2012, y más allá, hasta que la gente sienta de nuevo que estamos volviendo a los antiguos valores americanos en los que, si usted es un banquero, entonces usted está haciendo su dinero por medio de préstamos prudentes a hombres de negocios e individuos para que construyan plantas, así como equipos, $y$ contraten trabajadores que fabriquen bienes $y$ productos que levanten la economía y beneficien a todos (ibid: 8$)^{20}$.

Expresiones como "American Dream" (el Sueño Americano) o "old-fashioned American values" (los antiguos valores americanos); aunadas a imágenes como "gente levantándose y yendo a sus trabajos, fieles a sus empresas", representan una posición conservadora. Se trata de una apelación al pueblo.

Sin embargo, al aludir a reglas, normas y principios, dio cabida a que uno de los periodistas, Jake Taper, aprovechara para hacer una pregunta incómoda: ¿cuándo se responsabilizará a estos banqueros por no seguir las reglas? La respuesta inicialmente fue débil:

20 What I think is that the American people understand that not everybody has been following the rules; that Wall Street is an example of that; that folks who are working hard every single day, getting up, going to the job, loyal to their companies, that that used to be the essence of the American Dream. That's how you got ahead -- the old-fashioned way. And these days, a lot of folks who are doing the right thing aren't rewarded, and a lot of folks who aren't doing the right thing are rewarded.

And that's going to express itself politically in 2012 and beyond until people feel like once again we're getting back to some old-fashioned American values in which, if you're a banker, then you are making your money by making prudent loans to businesses and individuals to build plants and equipment and hire workers that are creating goods and products that are building the economy and benefitting everybody. 
Bien, primeramente, sobre el tema de las acciones judiciales en Wall Street, uno de los mayores problemas acerca del colapso de Lehmans y la posterior crisis financiera, así como el fiasco del conjunto de los préstamos hipotecarios, es que gran parte de todo esto no fue estrictamente ilegal, era inmoral, inapropiado o imprudente. Este es precisamente por lo que necesitamos aprobar a Dodd-Frank, para prohibir algunas de estas prácticas.

El sector financiero es muy creativo y ellos siempre están buscando formas para hacer dinero. Ese es su trabajo. Y si hay portillos $y$ reglas que puedan ser torcidas $y$ arbitrajes que puedan hacerse, ellos tomarán ventaja de esto. Así sin comentar acerca de procesos particulares - obviamente, este no es mi trabajo, es el trabajo del Fiscal General- yo pienso que de la frustración de la gente, parte de mi frustración, se deben a que un cúmulo de prácticas que no deberían haber sido permitidas no eran necesariamente acciones contra la ley, pero tuvieron un gran impacto destructivo. Y esto es porqué era importante para nosotros poner en acción reglas financieras que protejan al pueblo americano de decisiones imprudentes $y$ conductas irresponsables (ibid: 9$)^{21}$.

21 Well, first on the issue of prosecutions on Wall Street, one of the biggest problems about the collapse of Lehmans and the subsequent financial crisis and the whole subprime lending fiasco is that a lot of that stuff wasn't necessarily illegal, it was just immoral or inappropriate or reckless. That's exactly why we needed to pass Dodd-Frank, to prohibit some of these practices.

The financial sector is very creative and they are always looking for ways to make money. That's their job. And if there are loopholes and rules that can be bent and arbitrage to be had, they will take advantage of it. So without commenting on particular prosecutions - obviously that's not my job; that's the Attorney General's job - I think part of people's frustrations, part of my frustration, was a lot of practices that should not have been allowed weren't necessarily against the law, but they had a huge destructive impact. And that's why it was important for us to put in place financial rules
Al no tratarse de un comportamiento criminal (aunque inmoral y contra el espíritu de la normativa) no habría prisión. Sin embargo, la prisión no es la única sanción existente, puesto que ha habido un daño que debería ser reparado, aspecto sobre el cual nunca se cuestionó.

ows preocupó a muchos sectores, uno de ellos, American Bankers Association (ABA) recibió como propuesta para afrontar la situación siguiente:

\section{Investigación de la oposición}

ows tiene muchas de las características de una iniciativa bien financiada y los medios han especulado acerca de asociaciones con George Soros y otros. Será vital conocer quién está financiándolo $y$ cuáles son sus antecedentes y motivos. Si pudiéramos mostrar que ellos tienen las mismas motivaciones cínicas que su oponente político, eso debilitaría su credibilidad gravemente. Una meta estratégica sería, en definitiva, mostrar alguna evidencia de fraude en el otro bando.

Entregable: Nuestro trabajo de investigación de la oposición, en esta etapa, ofrecerá un análisis de quienes patrocinan y financian a ows, líderes extremistas, posiciones políticas $y$ una retórica para el desarrollo de encuestas y mensajería. La investigación también identificará las oportunidades para construir discursos negativos basados en hechos de ows para una difusión de alto impacto en los medios y así exponer a los patrocinadores detrás de este movimiento (Geduldig, Clark, Lytle y Cranferd, 2012: 3) ${ }^{22}$.

that protect the American people from reckless decision-making and irresponsible behavior.

22 Opposition Research.

ows bears many of the hallmarks of a well-funded effort and media reports have speculated about asociations with George Soros and others. It will be vital to understand who is funding it and what their backgrounds and motives are. If we can show they have the same cynical motivations as a politi- 
Este planteamiento surgió -en palabras de Larsen y Olshansky - una reconocida firma de lobbying en Washington, con vínculos en la industria de las finanzas (2011: 1) ${ }^{23}$.

Contratar servicios profesionales para el manejo de la imagen, asesoría retórica y demás es algo frecuente $y$ hasta recomendable en el ambiente político norteamericano. Sin embargo, la oferta de Clark Lytle Geduldig \& Cranford iba directamente enfocada a crear narrativas negativas (negative narratives). La ABA comunicó que en ningún momento se solicitó esa oferta (por ochocientos cincuenta mil dólares) y además, determinó no tomar ninguna acción relacionada con ella (Larsen y Olshansky, 2011:1).

Por último, es pertinente mencionar una de las consecuencias de todo este proceso: como parte de todo el fenómeno retórico suscitado en este contexto, surgió una doble retórica del temor. No solo es posible identificar un vector gubernamental en este sentido, sino también uno suscitado por el propio Occupy Wall Street.

En este sentido, para noviembre del 2011, durante la reunión celebrada en Florida de la Republican Governors Association (Asociación de Gobernadores Republicanos) con el propósito de definir políticas y estrategias de trabajo, el estratega republicano Frank Luntz, a quien ya se ha citado supra, manifestó: "Tengo mucho miedo ante este esfuerzo contra Wall Street. Estoy asustado por la muerte... Ellos están teniendo un impacto acerca de lo que el pueblo americano piensa del capitalismo" (Moody, 2011) ${ }^{24}$.

cal opponent it will undermine their credibility in a profound way. A key strategic goal will ultimately be to show any evidence of fraud on the other side. Deliverable: Our opposition research work at this stage will produce an analysis of OWS backers and funders, extremist leaders, policy positions, and rhetoric for the development of strategic polling and messaging. The research will also identify opportunities to construct fact-based negative narratives of the OWS for high impact media placement to expose the backers behind this movement.

23 a well-known Washington lobbying firm with links to the financial industry.

24 I'm so scared of this anti-Wall Street effort. I'm frightened to death... They're having an impact on what the American people think of capitalism.
Sus palabras parecieron sinceras, no dirigidas a producir algún tipo de situación efectista. Ciertamente, la magnitud, la duración y la novedad de Occupy Wall Street debió preocupar sobremanera no solo al gobierno norteamericano, sino a los mayores responsables de la crisis.

\section{BIBLIOGRAFÍA}

Arditi, Benjamín. "Las insurgencias no tienen un plan -ellas son el plan: performativos políticos y mediadores evanescentes en 2011". Debate Feminista 46. México, 2012.

Aristóteles . Retórica. Introducción, traducción y notas por Quintín Racionero. 2a reimpresión. Madrid: Editorial Gredos, 1999.

Brookings Institution. Not so demanding: why Occupy Wall Street need not make demands (yet). Last modified November 3, 2011.

Carney, Jay. Press gaggle by Press Secretary Jay Carney aboard Air Force One en route Asheville, NC. 17 de octubre de 2011. En: <http://www.whitehouse.gov/ the-press-office/2011/10/17/press-gagglepress-secretary-jay-carney-aboard-airforce-one-en-route-as $>$ [consultado el 6 de noviembre de 2011].

Carney, Jay. Press briefing by Press Secretary Jay Carney. 5 de octubre de 2011. En: $<$ http://www.whitehouse.gov/the-pressoffice/2011/10/05/press-briefing-presssecretary-jay-carney> [consultado el 5 de diciembre de 2011].

Carney, Jay. Press gaggle by Press Secretary Jay Carney and Deputy National Security Advisor for strategic communications Ben Rhodes. 15 de noviembre de 2011. En: <http://www.whitehouse.gov/thepress-office/2011/11/15/press-gagglepress-secretary-jay-carney-and-deputynational-security-adv> [consultado el 3 de abril de 2012].

Carney, Jay. Press briefing by Press Secretary Jay Carney. 30 de enero de 2012. En: $<$ http://www.whitehouse.gov/the-pressoffice/2012/01/30/press-briefing-presssecretary-jay-carney-13012> [consultado el 4 de diciembre de 2012]. 
Earnest, Josh. Press gaggle by Principal Deputy Press Secretary Josh Earnest en route Cincinnati, OH. 17 de setiembre de 2012. En: <http://www.whitehouse.gov/thepress-office/2012/09/17/press-gaggleprincipal-deputy-press-secretary-joshearnest-en-route-cinc> [consultado el 4 de diciembre de 2012].

Geduldig, Sam; Clark, Steve; Lytle, Gary y Cranford, Jay. Proposal. Occupy Wall Street Response. 2011. En: <http:// msnbcmedia.msn.com/i/msnbc/sections/ news/CLGF-msnbc.pdf> [consultado el 6 de mayo de 2012].

Hoffman, Meredith. "Protesters debate what demands, if any, to make". The New York Times. 16 de octubre de 2011. En: $<$ http://www.nytimes.com/2011/10/17/ nyregion/occupy-wall-street-trying-tosettle-on-demands.html> [consultado el 6 de noviembre de 2011].

Landers, Fred. "Occupy Wall Street, Urban Play, and the affirmation of crisis". Journal of Urban Culture Research 4. 2012. En: $<$ http://www.academia.edu/1997154/ Occupy_Wall_Street_Urban_Play_and_ the_Affirmation_of_Crisis> [consultado el 6 de diciembre de 2012].

Larsen, Jonathan y Olshansky, Ken. "Lobbying firm's memo spells out plan to undermine Occupy Wall Street". MSNBC TV. 19 noviembre de 2011. En: <http://investigations.nbcnews. com/_news/2011/11/19/8884405lobbying-firms-memo-spells-out-planto-undermine-occupy-wall-street $>$ [consultado el 6 de noviembre de 2012].

Moody, Chris. "How Republicans are being taught to talk about Occupy Wall Street". Yahoo! News. $1^{\circ}$ de diciembre de 2011. En: <http://news.yahoo.com/blogs/ticket/ republicans-being-taught-talk-occupywall-street-133707949.html> [consultado el 6 de abril de 2012].

Moore, Michael. Where Does Occupy Wall Street Go From Here? Michael Moore. com. 22 de noviembre 2011. En: <http:// www.michaelmoore.com/words/mikefriends-blog/where-does-occupy-wall- street-go-here $>$ [consultado el 26 de noviembre de 2011].

Mortara Garavelli, Bice. Manual de Retórica. 3era. edición. Madrid: Ediciones CátedraGrupo Anaya SA, 2000.

Obama, Barak. News Conference by the President. En: <http://www.whitehouse. gov/the-press-office/2011/10/06/newsconference-president> [consultado el 11 de junio de 2012].

Office of the Press Secretary of the White House. "Press briefing by Press Secretary Jay Carney". The White House. 5 de octubre de 2011. En: $<$ http://www.whitehouse.gov/the-pressoffice/2011/10/05/press-briefing-presssecretary-jay-carney>.

Office of the Press Secretary of the White House. "Press gaggle by Press Secretary Jay Carney aboard Air Force One en route Asheville, Nc". The White House. 17 de octubre de 2011. En: <http:// www.whitehouse.gov/the-pressoffice/2011/10/17/press-gaggle-presssecretary-jay-carney-aboard-air-forceone-en-route-as $>$.

Office of the Press Secretary of the White House. "Press gaggle by Press Secretary Jay Carney and deputy National Security Advisor for strategic communications Ben Rhodes. The White House. 15 de noviembre de 2011. En: <http:// www.whitehouse.gov/the-pressoffice/2011/11/15/press-gaggle-presssecretary-jay-carney-and-deputynational-security-adv>.

Office of the Press Secretary of the White House. "Press Briefing by Press Secretary Jay Carney". The White House. 30 de enero de 2012. En: $<$ http://www.whitehouse.gov/the-pressoffice/2012/01/30/press-briefing-presssecretary-jay-carney-13012>.

Office of the Press Secretary of the White House. "Press gaggle by Principal Deputy Press Secretary Josh Earnest en route Cincinnati, он". The White House. 17 septiembre de 2012. En: <http://www.whitehouse.gov/ the-press-office/2012/09/17/press-gaggle- 
principal-deputy-press-secretary-joshearnest-en-route-cinc>.

Perelman, Ch. y Olbrechts-Tyteca, L. Tratado de la argumentación. La Nueva Retórica. 1era. reimpresión. Madrid: Editorial Gredos SA, 1989.

Racionero, Quintín. Introducción, traducción y notas de 'Retórica' de Aristóteles. 2da. reimpresión. Madrid: Editorial Gredos, 1999.

Romanos, Eduardo. "El 15M y la democracia de los movimientos sociales". La vie des idees. 18 de noviembre de 2011. En: <http://www.booksandideas.net/ El-15M-y-la-democracia-de-los.html> [consultado el 6 de diciembre de 2012].

Romanos, Eduardo. "The strategic use of humor in the spanish indignado/15M
Movement". The Politics and Protest Workshop. CunY Graduate Center. 4 de octubre de 2012. En: <http:// politicsandprotest.ws.gc.cuny.edu/ files/2012/07/PPW-4-Romanos.pdf> [consultado el 6 de diciembre de 2012]. Taylor, Kate. "Bloomberg and Koch Deeply Split Over Blame on Fiscal Ills". The New York Times. 1 de noviembre de 2012. En: <http://www.nytimes. com/2011/11/02/nyregion/bloombergand-koch-split-over-blame-foreconomic-crisis.html> [consultado el 6 de noviembre de 2011].

Fecha de ingreso: 22/08/2013 Fecha de aprobación: 30/10/2013 
